\title{
The influence of a carbohydrate and whey protein based breakfast on metabolic and appetite parameters following a second meal
}

\author{
D. M. Allerton, M. D. Campbell, J. T. Gonzalez, D. J. West and E. J. Stevenson \\ Faculty of Health and Life Sciences, Northumbria University, Newcastle upon Tyne, NE1 8ST, UK
}

Whey protein consumption may improve metabolic health outcomes by influencing glucose metabolism, appetite and consequently energy balance ${ }^{(1)}$. Ingestion of whey has been shown to amplify insulin secretion in comparison with other proteins ${ }^{(2,3)}$ and this insulinotrophic property may be beneficial in reducing postprandial hyperglycaemia which, in the long term, is a significant risk factor for type 2 diabetes. The aim of the present study was to investigate the effect of adding whey protein to a carbohydrate breakfast on postprandial metabolism and appetite responses following a subsequent standard meal.

Healthy male participants ( $n=10$; age $24(2) \mathrm{y}$, mass $79.7(3.8) \mathrm{kg}$, BMI $24.5(2 \cdot 1) \mathrm{kg} / \mathrm{m}^{2}$ ) performed three trials in a randomised and counter-balanced fashion, consuming either a carbohydrate breakfast $(\mathrm{CHO})(1800 \mathrm{~kJ}, 86 \%$ energy from carbohydrate) with or without the addition of $20 \mathrm{~g}$ of whey protein isolate $(\mathrm{CHO}+\mathrm{WP})$, or omitting breakfast $(\mathrm{NB})$. At 180 minutes post-breakfast participants consumed a standardised pasta based lunch meal $(3427 \mathrm{~kJ}, 49 \%, 37 \%$ and $14 \%$ energy from carbohydrate, fat and protein respectively) and remained at rest for a further 180 minutes. Throughout, regular venous blood samples were collected for the determination of blood glucose, plasma insulin and plasma triglyceride. Visual analogue scales captured subjective appetite responses throughout the study protocol.

Blood glucose concentrations increased similarly after both breakfast meals (peak; CHO: $6 \cdot 44(0 \cdot 34)$ vs $\mathrm{CHO}+\mathrm{WP}: 5 \cdot 50(0 \cdot 17)$ $\mathrm{mmol} / 1, p>0.05)$, with no change observed under NB $(p>0.05)$. Post breakfast insulinaemia was greater after $\mathrm{CHO}+\mathrm{WP}$ than CHO (time averaged AUC; CHO: 154.7(18.5) vs CHO+WP: 193.1(26.3), $p=0.033$ ), while similar triglyceride responses were observed between all three trials $(p>0.05)$. Following a subsequent meal there were no differences across all trials in glycaemia (CHO: $3.99(0 \cdot 15)$ vs $\mathrm{CHO}+\mathrm{WP}: 4 \cdot 14(0 \cdot 13)$ vs NB: $4 \cdot 13(0 \cdot 96) \mathrm{mmol} / 1, p>0 \cdot 05)$ or insulinaemia $(\mathrm{CHO}: 136 \cdot 9(15 \cdot 7)$ vs $\mathrm{CHO}+\mathrm{WP}$ : $130 \cdot 7(18.8)$ vs NB: $110 \cdot 8(18 \cdot 6) \mathrm{pmol} / 1, p>0.05)$. Triglyceride concentrations were similarly elevated following lunch in $\mathrm{CHO}$ and $\mathrm{CHO}+\mathrm{WP}$ (AUC; CHO: $0 \cdot 99(0 \cdot 11)$ vs $\mathrm{CHO}+\mathrm{WP}: 1 \cdot 16(0 \cdot 16) \mathrm{mmol} / \mathrm{l}, p=0 \cdot 327$ ), both remaining significantly higher than NB $(0.82(0 \cdot 10) \mathrm{mmol} / 1, p>0 \cdot 05)$. There were no differences in sensations of fullness (AUC; CHO: 45(5) vs CHO+WP: 48(4) mm, $p>0.05$ ) or hunger (CHO: 54(4) vs $\mathrm{CHO}+\mathrm{WP}: 49(4) \mathrm{mm}, p>0.05)$ between $\mathrm{CHO}$ and $\mathrm{CHO}+\mathrm{WP}$ across the entire trial period (360 minutes).

The addition of whey protein to a carbohydrate-based breakfast increased the insulinaemic response to that meal, however it did not subsequently influence metabolic or appetite responses following a second meal. Omitting breakfast consumption induced comparable glycaemic, insulinaemic and appetite responses to subsequent feeding.

1. Sousa GT, Lira FS, Rosa JC et al. (2012) Lipids Health Dis 11, 67.

2. Gunnerud UJ, Ostman EM \& Björck IM (2013) Eur J Clin Nutr 67, 749-753.

3. Pal S \& Ellis V (2010) Br J Nutr 104, 1241-1248. 